\title{
Publisher's Note: Solar Irradiance Variability is Caused by the Magnetic Activity on the Solar Surface [Phys. Rev. Lett. 119, 091102 (2017)]
}

Kok Leng Yeo, Sami K. Solanki, Charlotte M. Norris, Benjamin Beeck, Yvonne C. Unruh, and Natalie A. Krivova

(Received 19 September 2017; published 29 September 2017)

DOI: 10.1103/PhysRevLett.119.139902

This paper was published online on 1 September 2017 with an omission in the funding statement on page 5. On page 5, right-hand column, the last paragraph before the references should read as "This work was supported by the German Federal Ministry of Education and Research (Project No. 01LG1209A), the European Research Council (ERC) under the European Union's Horizon 2020 research and innovation program (Grant Agreement No. 695075) and the BK21 plus program of the National Research Foundation (NRF) funded by the Ministry of Education of Korea." The paper has been corrected as of 19 September 2017. The funding statement is not present in the printed version of the journal. 